\title{
OPORTUNIDADES DE APLICAÇÕES DE BUSINESS INTELLIGENCE NO CONTEXTO DA INDÚSTRIA 4.0: REVISÃO SISTEMÁTICA DA LITERATURA 2015-2020
}

Versão do autor aceita publicada online: 22 maio. 2021

Publicado online: 30 jun. 2021

Como citar esse artigo - American Psychological Association (APA): Costa Neto, L. G. \& Campos, F. C. (2021). Oportunidades de aplicações de Business Intelligence no contexto da indústria 4.0: revisão sistemática da literatura 2015-2020. Exacta. DOI: https://doi.org/10.5585/exactaep.2021.19525.

\section{Luiz Gonzaga da Costa Neto}

gonzaga@gonzagaengenharia.com.br https://orcid.org/0000-0002-4566-0366

PPGEP/UNIMEP

Possui graduação em Engenharia Industrial Mecânica pela Universidade Metodista de Piracicaba (1989) e mestrado em Engenharia de Produção pela Universidade Metodista de Piracicaba (2006). Atualmente é diretor - Gonzaga Engenharia e Comércio de Manutenção Ltda e docente da Faculdade de Tecnologia de Piracicaba. Tem experiência na área de Engenharia de Produção, com ênfase em Gestão da Manutenção, atuando principalmente nos seguintes temas: confiabilidade, manutenção da qualidade, estratégia, performance, disponibilidade, manutenção preditiva através dos monitoramentos objetivo e subjetivo, e manutenção, estratégia, disponibilidade, segurança do trabalho.

\section{Fernando Celso de Campos}

fernando.campos@unimep.br

http://orcid.org/0000-0002-4900-8391

PPGEP/UNIMEP

Possui graduação em Ciência da Computação pelo Instituto de Ciências Matemáticas de São Carlos (ICMSC-USP) (1987), mestrado em Engenharia Mecânica pela Escola de Engenharia de São Carlos (EESC-USP) (1994), doutorado em Engenharia Mecânica pela Faculdade de São Carlos Engenharia (EESC-USP) (1999), pós-doutorado em Engenharia de Produção pelo DEP-UFSCar (2016). Atualmente é professor e pesquisador da Universidade Metodista de Piracicaba-UNIMEP atuando em cursos de graduação e pós-graduação. Tem experiência na área de Engenharia de Produção, com ênfase em Tecnologia da Informação Aplicada (sistema de informação, modelagem de processos, governança de TI, ERP, gestão do conhecimento), Tecnologias Digitais (Business Intelligence, BIA, Big Data Analysis, IoT), Green IT. Sustentabilidade (TI Verde, Economia Circular, Produção Mais Limpa), Indústria 4.0, Manutenção Industrial (PCM, Preventiva, Preditiva, Prescritiva, TPM), APL / gestão de cluster / modelos de cooperação, gestão de operações estratégicas e alternativas de projeto. Atuou na Engenharia de Métodos em projetos de melhoria contínua (Lean Manufacturing, SMED, Lean Office, Lean Healthcare, Gestão do uso de tecnologias na área da saúde).

\section{RESUMO}

A integração de sistemas de informação favorece a tomada de decisão que pode ter suporte das tecnologias de Business Intelligence, via relatórios e dashboards. Isso facilita a coleta de dados, análises e entrega de informações, para compor as bases decisórias da gestão de operações. No contexto da Indústria 4.0 o Business Intelligence conecta-se via internet of things. O objetivo desse artigo é identificar as oportunidades de aplicações de Business Intelligence na Indústria 


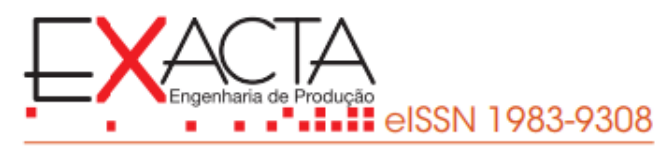

4.0. Para tanto, foi realizada uma Revisão Sistemática da Literatura via protocolo nas bases de dados: Scopus, Web of Science e Science Direct. Ao final, dezenove artigos selecionados, pelo critério do maior número de citações, identificaram onze tópicos principais. $\mathrm{O}$ impacto social vem pela demanda de profissionais melhor qualificados para Indústria 4.0. Em termos práticos a internet of things será usada massivamente para apoiar processos decisórios. Academicamente, esta síntese da literatura contribui para a melhor compreensão da temática.

Palavras-chave: Indústria 4.0; Business Intelligence; dashboard; IoT; qualificação profissional.

\section{ABSTRACT}

The integration of information systems favors decision making that can be supported by Business Intelligence technologies, via reports and dashboards. This facilitates data collection, analysis and delivery of information, to make up the decision-making bases of operations management. In the context of Industry 4.0 Business Intelligence connects via the Internet of things. The aim of this article is to identify the opportunities for Business Intelligence applications in Industry 4.0. For this, a Systematic Literature Review was performed via protocol in the databases: Scopus, Web of Science and Science Direct. At the end, nineteen selected articles, according to the criterion of the largest number of citations, identified eleven main topics. The social impact comes from the demand of better qualified professionals for Industry 4.0. In practical terms the internet of things will be used massively to support decisionmaking processes. Academically, this synthesis of literature contributes to a better understanding of the theme.

Keywords: Industry 4.0; Business Intelligence; dashboard; IoT; professional qualification.

\section{INTRODUÇÃO}

As indústrias buscam integrar seu sistema de uma forma capaz de alimentar os dados operacionais, sendo que, muitas organizações estão implementando tecnologias de Business Intelligence (BI) para relatórios de apoio e tomada de decisão. As tecnologias de BI facilitam os processos de coleta de dados, análise e entrega de informações, também chamados de Extraction, Transformation, Loading (ETL), ou seja, após essa transformação e limpeza dos dados, disponibilizam-se informações tratadas (carga) suficientemente para apoiar a tomada de decisão naquele assunto ou tema de negócio.

Goti et al. (2018) comentam que o surgimento de banco de dados, Big Data e Business Intelligence System (BIS), permitiram a criação de painéis de sistemas para medir o desempenho e gerenciar processos de negócios e, segundo Hanel e Felder (2017), juntos aos 


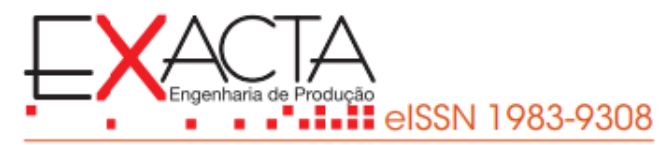

conceitos orientados para a engenharia, a aplicação de negócio de operação é discutida para apoiar decisões específicas de produção e organizar seus processos.

Coito et al. (2019) comentam que a iniciativa Indústria 4.0, manufatura digital, fábricas inteligentes, têm tudo a ver com as mudanças organizacionais introduzidas pelas tecnologias emergentes e dispositivos capazes de interagir autonomamente uns com os outros e com as pessoas. Essas expectativas podem ser atendidas pelo manufcturing execution system (MES) para melhorar o desempenho, a qualidade e a agilidade dos negócios de manufatura globalizados. E para Mantravadi e Moller (2019), além disso, o modelo da Indústria 4.0 também espera melhorar a produtividade atendendo as demandas por uma resposta em tempo real por meio do controle de processo.

O objetivo desse trabalho consiste na identificação das oportunidades de aplicações de BI no contexto da Indústria 4.0. Para tanto foi realizada uma RSL por meio das palavras-chave Indústria 4.0 e Business Intelligence, identificando os principais autores e periódicos mais relevantes.

$\mathrm{O}$ artigo está estruturado da seguinte maneira: seção 2 apresenta a Fundamentação Teórica relacionando Indústria 4.0, BI e a correlação entre esses conceitos. Seção 3 exibe o Método de Pesquisa utilizado no estudo. Seção 4 apresenta os Resultados dos dados coletados, sendo que a Análise e Discussão dos artigos selecionados são feitas na Seção 5. A Seção 6 traz os Achados e Tendências, e na Seção 7 são apresentadas as contribuições teóricas, práticas e para a sociedade.

Sendo as limitações por serem poucos os trabalhos encontrados com a temática analisada, com a questão de como as empresas aplicam as ferramentas de BI para dar suporte a tomada de decisão no contexto da indústria 4.0.

\section{INDÚSTRIA 4.0 e BUSINESS INTELLIGENCE}

Serão apresentados os conceitos da Indústria 4.0 e BI bem como sua possível ligação.

\subsection{Indústria 4.0}

Com os crescentes avanços nos processos e tecnologia de fabricação, o termo "Indústria 4.0" está se tornando um tópico cada vez mais importante. Este conceito apareceu em primeiro lugar em um artigo publicado em novembro de 2011 pelo governo alemão que resultou de uma iniciativa relativa à estratégia de alta tecnologia para 2020.

Schwab (2016) comenta que na Alemanha, há discussões sobre Indústria 4.0, um termo de 2011 na feira de Hannover para descrever como isso iria revolucionar a organização das cadeias globais de valor. Ao permitir fábricas inteligentes, a quarta revolução industrial cria um mundo no qual os sistemas físicos e virtuais de fabricação cooperam de forma global e flexível, 


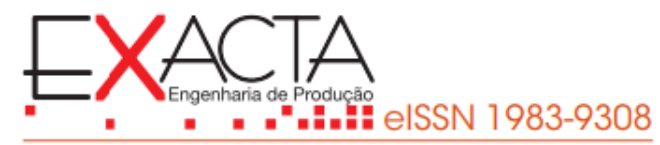

permitindo total personalização de produtos e a criação de novos modelos operacionais. Odważny et al. (2019) comentam que dentro deste período desde 2011, vários modelos, ideias e tecnologias foram desenvolvidas, por exemplo: fábrica inteligente, sendo as tecnologias habilitadoras com maior impacto à indústria e para a sociedade: Internet of Things (IoT), Internet of Services (IoS), cloud computing e cyber-physical systems (CPS), complementado por Robótica, Big Data, Manufatura de Nuvem e Realidade Aumentada (PEREIRA e ROMERO, 2017).

\subsection{Business Intelligence}

Para Barbieri (2001) BI pode ser entendido como a "utilização de várias fontes de informação para definir estratégias de competitividade nos negócios da empresa", podendo ser incluídos nessa definição os conceitos de estruturas de dados, representadas pelos bancos de dados tradicionais, data warehouse e data marts, criados objetivando-se o tratamento relacional e dimensional de informações, bem como as técnicas de data mining aplicadas sobre elas, buscando-se correlações e fatos "escondidos".

Segundo Odważny et al. (2019) um dos principais recursos de desenvolvimento é o uso de BI na indústria, permitindo reunir e analisar dados coletados e complexos de processos operacionais em cada nível da organização, podendo o usuário ter a possiblidade de ver a situação de cada recurso a qualquer momento desde o início da operação.

De acordo com Aqel, Nakshabanda, Adeniyi (2019), os avanços no uso de BI e Big Data, levará a introdução de novos aplicativos e métodos para expandir a capacidade do Decision Support System (DSS) já existentes.

\subsection{Indústria 4.0 e Business Intelligence}

A Indústria 4.0 e o BI estão interligados por meio da conectividade da IoT, sendo que Garcia e Garcia (2019) comentam que no contexto da Indústria 4.0 as empresas de manufatura identificam oportunidades de desenvolver sua competitividade em suas operações organizacionais pela análise de dados, e o BI está focado principalmente na aquisição e análise de informações, segundo Olszak e Mach-krol (2018). De acordo com Goti et al. (2018) o BI se torna um banco de dados, permitindo a criação de painéis de sistema para medir o desenvolvimento e gerenciar os processos de negócios.

As tecnologias por meio da IoT que facilitam os processos de coleta de dados, aliadas aos esquemas ETL desses dados, favorecem a análise e entrega de informações disponibilizandoas para apoiar a tomada de decisão nas operações de negócios empresariais, evidenciando oportunidades de aplicações de ferramentas de BI no contexto da Indústria 4.0. 


\section{MÉTODO DE PESQUISA}

O método de pesquisa adotado para se atingir o objetivo deste trabalho foi a partir de uma RSL que é um processo científico, permitindo avaliação da literatura, por meio da identificação da seleção e da avaliação dos estudos existentes (TRANFIELD, DENYER, ESMART, 2003). Diferente da revisão teórica tradicional, este método pode ser replicado por outros pesquisadores (SAYFOURI, 2014). Para Reim, Parida, Ortqvistet (2015), a revisão sistemática da literatura torna as conclusões mais elaboradas, auxiliando o desenvolvimento de novos horizontes teóricos.

\subsection{Protocolo da RSL}

Essa RSL foi realizada com suporte do Software StArt - State of the Art through Systematic Review, versão 3.4 Beta - LAPES - Laboratório de Pesquisa em Engenharia de Software UFSCAR (StArt, 2020).

O protocolo de pesquisa é apresentado no Quadro1, com todos os elementos utilizados.

Quadro 1: Protocolo de Pesquisa

\begin{tabular}{|c|c|}
\hline Estratégia & Protocolo \\
\hline $\begin{array}{l}\text { Questão da } \\
\text { pesquisa }\end{array}$ & $\begin{array}{l}\text { Como as empresas aplicam as ferramentas de BI para dar suporte à } \\
\text { tomada de decisão no contexto da Indústria } 4.0 \text {. }\end{array}$ \\
\hline Base de dados & Scopus, Web of Science, Science Direct \\
\hline String de busca & "Industry 4.0" And "Business Intelligence" \\
\hline Período & 2015 a Setembro/2020 \\
\hline Idioma & Inglês, Português, Espanhol \\
\hline & $\begin{array}{l}\text { - Artigos duplicados. } \\
\text { de busca. } \\
\text { - Artigos publicados fora do período. } \\
\text { - Livros, Sites e Teses. }\end{array}$ \\
\hline $\begin{array}{l}\text { Critérios de } \\
\text { inclusão }\end{array}$ & $\begin{array}{l}\text { - Artigos que contenham o título, resumo e palavras chave as } \\
\text { strings de busca. } \\
\text { - Artigos publicados no período }\end{array}$ \\
\hline
\end{tabular}
Fonte: Autores

\subsection{Pesquisas Iniciais e Refinamentos}

A pesquisa inicial resultou num total de 165 trabalhos, incluindo somente artigos no período de 2015 a setembro/2020, sendo assim distribuídos: 99 (60\%) da base de dados Scopus, 61 (37\%) da base de dados Science Direct e $5(3 \%)$ da base de dados Web of Science. 
Foram realizados três processos de filtragem das pesquisas iniciais para selecionar os artigos a serem avaliados:

$\checkmark$ Primeiro filtro: Exclusão dos títulos não relacionados com o objetivo da pesquisa, artigos duplicados, artigos que não contenham no título, resumo e palavras chave as strings de busca, artigos publicados fora do período, livros, sites e teses, resultando em 122 artigos rejeitados e 3 artigos duplicados.

$\checkmark$ Segundo filtro: Inclusão dos artigos que continham no título, resumo e palavras chaves, a string de busca, artigos publicados no período de 2015 a setembro/2020, nos idiomas Inglês, Português, Espanhol, resultando em 40 artigos.

$\checkmark$ Terceiro filtro: Constituiu na leitura dos resumos e das conclusões desses 40 artigos, com o intuito de selecionar somente os artigos que tratam o objetivo da pesquisa, resultando em 19 artigos para análise.

\section{RESULTADOS}

Serão apresentadas as análises dos dados dos 19 artigos selecionados: por ano de publicação, por periódicos, por citações.

Do ano de 2017, o primeiro artigo foi publicado no Procedia, na 10th CIRP Conference on Intelligent Computation in Manufacturin Engineering, por Hanel e Felden (2017) da Technische University Bergakadenie Freiberg - Alemanha, cujo foco do artigo, seria os conceitos orientados para engenharia, a aplicação de inteligência artificial de negócios e discutida para apoiar decisões específicas e organizações dos processos, e o segundo publicado na 27th Intertational Conference on Flexible Automation na Intelligent Manufacturin, por Benesova e Tupa (2017) da University of West Bohemia in Pilsen e do Departament of Tecnologies and Measuprement - República Checa, indicando no artigo que com o advento da Indústria 4.0 e suas tecnologias emergentes, existirá a necessidade de trabalhador treinado e certificado na área de computação, algoritmos de autoaprendizagem e análise de dados.

A Tabela 1 apresenta os periódicos que mais publicaram sobre o tema: Sustainability com 4, Procedia Manufacturing com 3 e Applied Science com 2 artigos publicados. Esses três periódicos juntos foram responsáveis por 9 publicações dos 19 artigos selecionados. 
Tabela 1: Periódicos com números de publicações

\begin{tabular}{lcc}
\hline Periódico & $\begin{array}{c}\text { Fator de } \\
\text { Impacto } \\
\mathbf{( 2 0 1 9 )}\end{array}$ & $\begin{array}{c}\text { Número de } \\
\text { publicação }\end{array}$ \\
\hline Applied Sciences & 2,474 & 02 \\
\hline Procedia Manufacturing & 1,590 & 03 \\
\hline Sustainability & 0,581 & 04 \\
\hline Total & & $\mathbf{0 9}$ \\
\hline
\end{tabular}

Fonte: Autores

A Análise de Citações demonstra a quantidade de vezes que o trabalho foi citado ao longo do tempo, sendo apresentados na Tabela 2 os artigos com maior número de citações, identificando ao todo 19 autores nos seis artigos mais citados (Top Six).

O artigo com maior número de citações é o de Benesova e Tupa (2017) com 274 citações, que tratou da relevância da identificação de cargos nas empresas, devido ao advento da Indústria 4.0 necessitar de profissionais treinados e qualificados para essa nova era da tecnologia.

Seguido pelo artigo de Nagy et al. (2018), com 182 citações, tratando de como as empresas que operam na Hungria interpretam o fenômeno da Indústria 4.0, e quais as ferramentas da IoT usam para apoiar seus processos e quais problemas críticos enfrentam durante a adaptação.

Tabela 2: Artigos mais citados (Top Six)

\begin{tabular}{|c|c|c|}
\hline Autor (Ano) & Título do Artigo & $\begin{array}{l}\text { Número de } \\
\text { citações }\end{array}$ \\
\hline Benesova e Tupa (2017) & $\begin{array}{c}\text { Requirements for Education and } \\
\text { Qualification of People in Industry } 4.0\end{array}$ & 274 \\
\hline Nagy et al. (2018) & $\begin{array}{l}\text { The role and impact of industry } 4.0 \text { and } \\
\text { the internet of things on the business } \\
\text { strategy of the value chain-the case of } \\
\text { hungary }\end{array}$ & 182 \\
\hline $\begin{array}{l}\text { Ruppert, T., Jasko, S., } \\
\text { Holczinger, T.Abonyi, J. } \\
(2018)\end{array}$ & $\begin{array}{l}\text { Enabling technologies for operator 4.0: A } \\
\text { survey }\end{array}$ & 53 \\
\hline $\begin{array}{c}\text { Ansari, F., Glawar, R., } \\
\text { Nemeth, T. } \\
\text { (2019) }\end{array}$ & $\begin{array}{l}\text { PriMa: a prescriptive maintenance model } \\
\text { for cyber-physical production systems }\end{array}$ & 23 \\
\hline $\begin{array}{l}\text { Olszak, C. M. Mach-Krol, M. } \\
\text { (2018) }\end{array}$ & $\begin{array}{l}\text { A conceptual framework for assessing an } \\
\text { organization's readiness to adopt big data }\end{array}$ & 19 \\
\hline $\begin{array}{l}\text { Mantravadi, Soujanya ; } \\
\text { Moller, Charles (2019) }\end{array}$ & $\begin{array}{l}\text { An Overview of Next-generation } \\
\text { Manufacturing Execution Systems: How } \\
\text { important is MES for Industry 4.0? }\end{array}$ & 15 \\
\hline
\end{tabular}

Os próximos quatro artigos mais citados apresentados na Tabela 2, vão tendo temas envolvendo tecnologias habilitadoras, modelo de manutenção prescritiva para sistemas CPS, um framework que checa a prontidão em adotar Indústria 4.0 a partir de análises de Big Data, e da aplicação de Manufacturing Execution Systems (MES), verificando de forma abrangente em termos de 
grau de importância para releases de próxima geração de família de sistemas, que abrem possibilidades da interação do BI nesses contextos.

\section{ANÁLISE E DISCUSSÃO}

Para a análise e discussão parte-se do Quadro 2, que apresenta uma síntese da amostra dos 19 artigos selecionados e os principais tópicos que eles abordam e são destacados nesse momento.

O Quadro 2 está organizado por ordem alfabética de autores.

Quadro 2: Síntese da amostra dos artigos

\begin{tabular}{|c|c|}
\hline Autor (ano) & Temas destacados \\
\hline Ahmad et al. (2020) & $\begin{array}{l}\text { Business Intelligence System (BIS) é uma combinação de } \\
\text { ferramentas técnicas para apoiar a tomada de decisão. }\end{array}$ \\
\hline Ansari, Glawar, Nemeth (2019) & Abordagem integrada da Prescriptive Maintenance (PriMa). \\
\hline Aqel, Nakshabanda, Adeniyi (2019) & $\begin{array}{l}\text { Avanços no uso de Big Data, novos aplicativos e métodos para } \\
\text { expandir a capacidade do Decision Support System (DSS). }\end{array}$ \\
\hline Benesova e Tupa (2017) & $\begin{array}{l}\text { Necessidade de trabalhadores qualificados e certificados nas } \\
\text { áreas de computação, algoritmos de autoaprendizagem e análise } \\
\text { de dados. }\end{array}$ \\
\hline Cao, Cai, Yue (2019) & $\begin{array}{l}\text { Mobile Wireless Sensor Networks (WMSNs)), transmitindo } \\
\text { continuamente dados percebidos para a estação base na forma de } \\
\text { comunicação sem fio. }\end{array}$ \\
\hline Coito et al. (2019) & $\begin{array}{l}\text { Qualidade dos Dados Coletados: poluídos, incompletos e } \\
\text { ausentes, necessitando de tratamento adequado. }\end{array}$ \\
\hline Eggert e Alberts (2020) & $\begin{array}{l}\text { Anos } 1990 \text { o termo Big Data nasce com mudança de dados em } \\
\text { volume, velocidade, variedade e veracidade. }\end{array}$ \\
\hline Garcia e Garcia (2019) & Tratamento das tecnologias emergentes da Indústria 4.0. \\
\hline Goti et al. (2018) & $\begin{array}{l}\text { Sistema de banco de dados, Big Data, painéis para medir } \\
\text { desenvolvimento gerencial, sistema de produção "X" (XPS). }\end{array}$ \\
\hline (2019) & $\begin{array}{l}\text { Programa de Condition Based Maintenance (CBM) em um } \\
\text { ambiente industrial, com o objetivo de determinar os valores } \\
\text { ótimos dos limites de intervenção preventiva em equipamentos } \\
\text { sob critérios de custo de manutenção corretiva e preventiva. }\end{array}$ \\
\hline Han & $\begin{array}{l}\text { Uso do sistema de TI para enriquecer o cumprimento de tarefas } \\
\text { com informações. }\end{array}$ \\
\hline renda, Bertoncelj (20 & $\begin{array}{l}\text { Capacidade de influenciar a melhoria dos processos por meio de } \\
\text { sua própria otimização em tomada de decisão autônoma, com } \\
\text { sensores conectados. }\end{array}$ \\
\hline di e Moller (2019) & $\begin{array}{l}\text { Enterprise Information System (EIS), hardware e software, } \\
\text { processo de dados e pessoas. }\end{array}$ \\
\hline Nagy et al. (2018) & Digitalização industrial, Big Data, RFID, soluções diversas. \\
\hline Odważny et al. (2019) & $\begin{array}{l}\text { Ferramentas de Information Technology (TI) no ambiente } \\
\text { industrial, transformação de dados em informações claras e } \\
\text { análise para apoiar a decisão. }\end{array}$ \\
\hline Olszak e Mach-krol (2018) & $\begin{array}{l}\text { Big Data, Competitive Intelligence (CI), evolução na análise de } \\
\text { sistema. }\end{array}$ \\
\hline Ruppert et al. (2018) & $\begin{array}{llll}\text { Sensores inteligentes, dispositivos } & \text { vestíveis, gerando } \\
\text { comunicação de dados. }\end{array}$ \\
\hline Saglain (2019) & IoT é a rede global de dispositivos físicos inter-relacionados. \\
\hline Soni et al. ( 2020) & Dispositivos e o impacto socio econômico. \\
\hline
\end{tabular}

Fonte: Autores.

A partir do conteúdo do Quadro 2 foram analisados os temas abordados em agrupamentos na seguinte sequência: ferramentas e sistema de TI, qualidade dos dados coletados, tipos de 


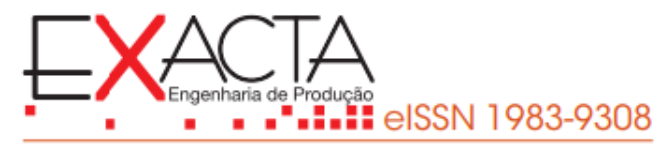

sistemas (EIS, DSS, BIS), rede para coleta e análise de dados, aplicação de IoT, sensores inteligentes e dispositivos vestíveis, Big Data, Ind.4.0 e BI, aplicações na área de manutenção, fábrica inteligente, possibilitando impactar não só as pesquisas como também o mercado de trabalho, promovendo oportunidades de novos modelos de negócios e suas aplicações alterando as relações da sociedade com as tecnologias.

De acordo com Odważny et al. (2019), os conceitos de desenvolvimento de ferramentas de Information Technology (TI) no ambiente industrial, podem ser descritos como aqueles que transformam pilhas de dados em informações claras e análise para apoiar a decisão, definidos como BI, permitindo reunir e analisar dados complexos de processos operacionais em cada nível da organização, com possiblidade de ver a situação de cada recurso a qualquer momento desde o início da operação. Para Hanel e Felden (2017) os tomadores de decisão na indústria usam sistema de TI para enriquecer o cumprimento de tarefas com informações de forma sistemática e a aplicação de BI é discutida para apoiar decisões específicas de produção e para organizar seus processos, sendo capaz de automatizar a integração e análise de dados de produção em favor de um suporte multidimensional à decisão.

Destaque à qualidade dos dados coletados que, para Coito et al. (2019), às vezes vem poluídos, incompletos ou complementarmente ausentes, com muitos erros, e no campo da ciência de dados e BI, os dados devem ser tratados de maneira adequada para não tornar um gargalo para o desenvolvimento da indústria. Esse é um ponto de atenção importante.

Três trabalhos destacaram alguns tipos de sistemas: EIS, DSS e BIS, e são detalhados a seguir. Mantravadi e Moller (2019) destacam que o Enterprise Information System (EIS), composto de hardware, software, processo de dados e pessoas, podem ser classificados em seis tipos, um deles é o BI. Nos últimos anos pode-se observar o surgimento de muita proposta e serviços inteligentes, seus dispositiyos e o impacto sócio econômico (SONI et al., 2020) e para Aqel, Nakshabanda, Adeniyi (2019), os avanços no uso de BI e Big Data, levarão à introdução de novos aplicativos e métodos para expandir a capacidade do Decision Support System (DSS) já existente. O DSS, inclusive tem a capacidade de mineração de dados de forma eficaz e estruturada para que possa ser usado para apoio à tomada de decisão nas organizações.

Segundo Ahmad et al. (2020) estudos anteriores focaram apenas na perspectiva da utilização de Big Data na Indústria 4.0 e negligenciaram o papel do Business Intelligence System (BIS). O BIS é uma combinação de ferramentas técnicas que fornecem informações históricas para seus usuários para análise, consulta e relatório que apoiam o gerenciamento e a tomada de decisão que significa aumentar a eficiência dos processos de negócios, permitindo que não apenas projetem a competitividade, mas, aumenta a receita, sendo que, os pesquisadores e 


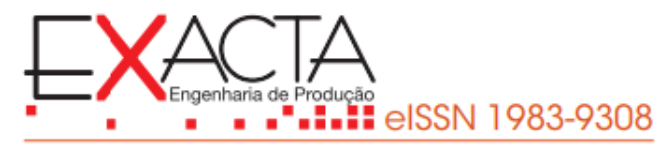

profissionais endossam que as tecnologias de BI alcançaram notáveis considerações de ambos, organizações e indústrias. Pesquisa de Gartner Group confirmaram quase 80\% das empresas nos Estados Unidos da América e 50\% das empresas Europeias integram o BIS (AHMAD et al., 2020).

Nagy et al. (2018) comentam que na era da digitalização industrial, as empresas estão cada vez mais investindo em ferramentas e soluções que permitam que seus processos, máquinas, funcionários e aos próprios produtos sejam integrados em uma única rede para coleta e análise de dados. Ferramentas que geram dados e criam Big Data são essenciais, incluindo, sensores, chips por Radio Frequency Identification (RFID), impressora 3D, câmeras e robôs. Máquinás e pessoas usam interfaces para se comunicar na maioria das vezes em tempo real, e ferramentas adicionais como nuvens, dados locais, armazéns e Enterprise Resource Planning (ERP) coletam, armazenam e distribuem dados.

Saglain (2019) pesquisando acerca do ambiente industrial afirma que a IoT é a rede global de dispositivos físicos inter-relacionados como sensores, atuadores, aplicativos inteligentes, objetos, dispositivos de computação, máquinas mecânicas e pessoas, e que está se tornando a fonte de dados e informações abundantes nos processos de fabricação, trazendo desafios substancias para a coleta, processamento e tomada de decisão em tempo real. Destaque-se também o trabalho de Ruppert et al. (2018) comentando que os sensores inteligentes $\boldsymbol{e}$ dispositivos vestíveis, proporcionam a oportunidade para desenvolver espaços de trabalho inteligentes para operadores, como os sistemas Humanos - Ciber-físicos, integrando-os em processos de fabricação flexíveis e multifuncionais, sendo que o BI é um banco de dados para uma tomada de decisão por meio de comunicações destes sensores e dispositivos.

Eggert e Alberts (2020) afirmam que desde o início da análise de dados nos anos 1950, os pesquisadores tinham interesse em desenvolver novos métodos para fornecer intuições sobre dados usando ferramentas de BI que permitem produzir e capturar uma grande quantidade de dados, sendo que até o início dos anos 1990, dados estruturados, como dados numéricos em tabelas dominaram a área de análise de dados. Após esta primeira etapa, a chegada de dados não estruturados com vídeo, música ou arquivo de texto, levou um aumento exponencial de dados a serem analisados e o termo Big Data nasce descrevendo a mudança de dados em volume, velocidade, variedade e veracidade, e a terceira onda de análise de dados começou com novas fontes de dados como dispositivos moveis e sensores sem fio conectados, permitindo oportunidades avançadas de coleta e análise de dados. 


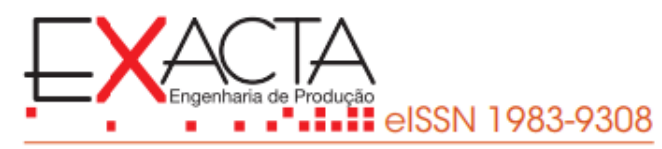

Olszak e Mach-krol (2018) comentando sobre Big Data e questões de sustentabilidade, lembram as premissas básicas que fundamentam a ideia de BI e Competitive Inlelligence (CI): necessidade de dados tratados conforme um padrão desejado de análise.

Para Goti et al. (2018) o sistema de banco de dados, Big Data e BI, permitem a criação de painéis (ou dashboards) para medir e gerenciar os processos de negócios e a avaliação da implementação dos princípios, métodos e ferramentas do XPS - Sistema de Produção "X".

A Indústria 4.0 está atualmente transformando a sociedade e mudando os setores agrícola, industrial e de serviços. Neste contexto, as empresas de manufaturas identificaram como oportunidade de desenvolver sua competitividade em suas operações e eficiênciás organizacionais (GARCIA E GARCIA, 2019). Os autores apresentaram as implicações dos novos recursos das tecnologias centrais e de suporte da Indústria 4.0. Entre as tecnologias destacadas, a análise de dados $\boldsymbol{e}$ inteligência artificial, tanto no gerenciamento de produção como de manutenção com um nível de impacto médio e alto em quase todas as tarefas de planejamento. Isto fortalece a necessidade das empresas tratarem esta tecnologia para a possibilidade de BI com a tarefa de planejamento.

Dentro do aspecto de manutenção, os programas Condition Based Maintenance (CBM), segundo Goti et al. (2019), podem fornecer vantagens significativas com o objetivo de determinar o ponto potencial de falha, que possibilitará a intervenção preventiva para equipamentos. Um modelo matemático foi desenvolvido pelo autor, sob custo de manutenção corretiva e critério de preventivá, considerando evolução em qualidade e velocidade de produção juntamente com a manutenção corretiva e a manutenção preventiva baseada nas condições. Este modelo poderá fornecer dados para tomada de decisão por meio de BI. A abordagem integrada da Prescriptive Maintenance (PriMa) aprimora as duas capacidades funcionais, sendo a primeira com processamento eficiente com grande quantidade de dados multimodais e heterogêneos coletados de fonte de dados multidimensionais e a segunda gerar medidas de apoio e recomendações para melhorar e otimizar os próximos planos de manutenção correlacionados com o PCP - Planejamento e Controle de Produção (ANSARI, GLAWAR, NEMETH, 2019). Para Cao, Cai, Yue (2019) a coleta de dados é uma das principais tarefas de Mobile Wireless Sensor Networks (WMSNs)), transmitindo continuamente dados coletados para a estação base na forma de comunicação sem fio. Uma boa fonte de dados para a tomada de decisão, porém enfrenta muitos desafios por causa dos limites de energia, poder de computação e capacidade de armazenamento do sensor, bem como a influência da interferência do canal e a mutação do ambiente. 
De acordo com Jerman, Erenda, Bertoncelj (2019) os elementos cruciais de uma fábrica inteligente como funcionários, máquinas e produtos têm um efeito importante nos modelos de negócios atuais, sendo que, sua produção e equipamento com sensores em sistemas autônomos gera a capacidade de influenciar a melhoria dos processos.

Por fim, o trabalho de Benesova e Tupa (2017) destaca que a indústria foi afetada por mudanças tecnológicas e inovações, chamadas de revoluções, não influenciando apenas na produção, mas também o mercado de trabalho e o sistema educacional.

Com o advento da Indústria 4.0 e suas tecnologias habilitadoras, existirá a necessidade de trabalhadores bem mais capacitados e bem mais qualificados nas áreas de computação, algoritmos de autoaprendizagem e análise de dados, e o BI é o painel em que isso se materializa.

\section{ACHADOS E TENDÊNCIAS}

O Quadro 3 apresenta as ferramentas de BI, utilizadas na coleta de dados para informações e tomada de decisão no contexto da Indústria 4.0, com destaque para o Big Data e Sensores sem fio com três ocorrências.

Quadro 3: Ferramentas de BI

\begin{tabular}{|l|c|c|c|c|c|}
\hline \multicolumn{1}{|c|}{ FERRAMENTAS } & $\mathbf{2 0 1 7}$ & $\mathbf{2 0 1 8}$ & $\mathbf{2 0 1 9}$ & $\mathbf{2 0 2 0}$ & $\mathbf{N}^{\mathbf{0}}$ Ocorrência \\
\hline Big Data & & $\mathrm{X}$ & $\mathrm{X}$ & $\mathrm{X}$ & 3 \\
\hline Dispositivos móveis & & & & $\mathrm{X}$ & 1 \\
\hline Sensores sem fio & & $\mathrm{X}$ & $\mathrm{X}$ & $\mathrm{X}$ & 3 \\
\hline Inteligência artificial & & & $\mathrm{X}$ & $\mathrm{X}$ & 2 \\
\hline CBM - Condition Based Maintenance & & & $\mathrm{X}$ & & 1 \\
\hline PriMa - Prescriptive Maintenance & & & $\mathrm{X}$ & & 1 \\
\hline DSS - Decision Support System & & & $\mathrm{X}$ & & 1 \\
\hline Máquinas & & & $\mathrm{X}$ & & 1 \\
\hline Pessoas & & & $\mathrm{X}$ & & 1 \\
\hline EIS - Enterprise Information System & $\mathrm{X}$ & & $\mathrm{X}$ & & 2 \\
\hline TI - Information Technology & $\mathrm{X}$ & & $\mathrm{X}$ & & 2 \\
\hline IoT - Internet of Things & $\mathrm{X}$ & & & & 1 \\
\hline Qualificação dos profissionais & $\mathrm{X}$ & $\mathrm{X}$ & & & \\
\hline Tecnologias emergentes da Indústria 4.0 & & $\mathrm{X}$ & & & \\
\hline ERP - Enterprise Resource Planning & & $\mathrm{X}$ & & & \\
\hline Dispositivos vestíveis & & & $\mathrm{X}$ & & 1 \\
\hline MES - Manufacturing Execution System & & $\mathrm{X}$ & & & 1 \\
\hline BI\&A - Business Intelligence and Analytics & & $\mathrm{X}$ & & $\mathrm{X}$ & 2 \\
\hline XPS - Sistema de Produção "X" & & & 1 \\
\hline RFID - Radio frequency identification & & $\mathrm{X}$ & \\
\hline
\end{tabular}

Fonte: Autores.

Observa-se no Quadro 3 que as ferramentas apresentadas para o uso do BI, passaram a ser fortemente destacadas a partir de 2018, sendo que, o Big Data e sensores sem fio aparecem nas 


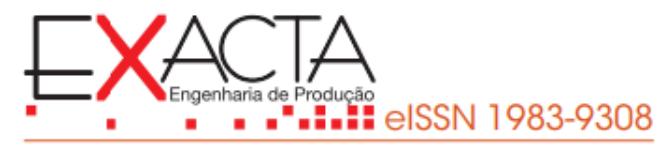

publicações de três anos consecutivos. O destaque é que o BI além de um banco de dados poderá fornecer dados para tomada de decisão em ambiente operacional, por meio de comunicações dos sensores inteligentes e dispositivos vestíveis. A coleta de dados é uma das principais tarefas de Mobile Wireless Sensor Networks (WMSNs), mas enfrenta muitos desafios: limites de energia, poder computacional/processamento e capacidade de armazenamento de dados, influência da interferência do canal e a mutação do ambiente.

Os processos e conceitos de desenvolvimento de ferramentas TI no ambiente industrial, transformam os dados em informações claras e os tomadores de decisão na indústria usam sistemas de TI com essas informações de forma sistemática, para apoiar decisões específicas de produção e para organizar seus processos, gerando possibilidades de diferenciais de competitividade.

Com o advento da Indústria 4.0 e suas tecnologias habilitadoras, existirá a necessidade de trabalhadores bem mais capacitados e bem mais qualificados nas áreas de computação, algoritmos de autoaprendizagem e análise de dados.

\section{CONCLUSÃO}

O objetivo deste trabalho de buscar oportunidade de aplicações de BI no contexto da Indústria 4.0, foi atendido ao se identificar um total de 19 artigos selecionados e analisados. No tocante à teoria, a RSL contribuiu para verificar a situação atual da literatura em relação à abordagem do uso de BI no ambiente da Indústria 4.0, além de apontar os trabalhos mais relevantes para análise, entendimento e aprofundamento do assunto. Foi possível observar que poucos trabalhos publicados abordam a temática analisada, visto que a nuvem de palavras chaves resultante do conjunto de artigos contempla mais a Indústria 4.0 e informações de dados pela ferramenta Big Data. Obserya-se que, na prática existe necessidade de utilização do BI nas organizações para apoiar a tomada de decisão, isso se torna possível em função das tecnologias já existentes. Os trabalhos analisados apresentam aplicações de ferramentas da tecnologia emergente da Indústria 4.0 como BI\&A e IoT, ou seja, o BI com outros tipos de interface.

As profundas mudanças no setor industrial apresentam fortes impactos ao longo de toda a cadeia de valor surgindo, novas tecnologias, criação de novos empregos e nova forma da organização de trabalho, assim o BI também está passando por essa transformação, proporcionando à sociedade novas oportunidades de modelos de negócios.

A revisão realizada apresenta as seguintes limitações: foram encontrados os trabalhos com a temática analisada, e por serem poucos, os critérios de seleção e filtragens foram pautados nas palavras chaves. Apesar das limitações apresentadas, o artigo contribuiu para a literatura, com escopo de atuação que o tema possibilitou. 


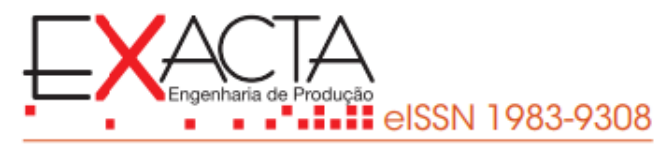

Como sugestões para pesquisas futuras: utilizar a combinação das palavras chaves buscando um cenário mais abrangente de publicações, como também, propor a utilização de BI para tomada de decisão nos indicadores de manutenção.

\section{REFERÊNCIAS}

Ahmad, S., Miskon, S., Alabdan, R., \& Tlili, I. (2020). Towards Sustainable Textile and Apparel Industry: Exploring the Role of Business Intelligence Systems in the Era of Industry 4.0. Sustainability, 12(7), 2632. https://doi.org/10.3390/su12072632

Ansari, F., Glawar, R., \& Nemeth, T. (2019). PriMa: a prescriptive maintenance model for cyber-physical production systems. International Journal of Computer Integrated Manufacturing, 32(4-5), 482-503. https://doi.org/10.1080/0951192X.2019.1571236

Aqel, M. J., Nakshabandi, O. A., \& Adeniyi, A. (2019). Decision support systems classification in industry. Periodicals of Engineering and Natural Sciences, 7(2), 774-785. DOI: http://dx.doi.org/10.21533/pen.v7i2.550

Benešová, A., \& Tupa, J. (2017). Requirements for education and qualification of people in $\begin{array}{lllll}\text { Industry } & 4.0 . & \text { Procedia } & \text { Manufacturing, } & 11,\end{array}$ https://doi.org/10.1016/j.promfg.2017.07.366

Barbieri C. (2001). BI - Business Intelligence: modelagem \& tecnologia. Rio de Janeiro: Axcel Books, 424p.

Cao, L., Cai, Y., \& Yue, Y. (2019). Swarm Intelligence-Based Performance Optimization for Mobile Wireless Sensor Networks: Survey, Challenges, and Future Directions. IEEE Access, 7, 161524-161553. DOI: 10.1109/ACCESS.2019.2951370

Coito, T., Viegas, J. L., Martins, M. S., Cunha, M. M., Figueiredo, J., Vieira, S. M., \& Sousa, J. M. (2019). A Novel Framéwork for Intelligent Automation. IFAC-PapersOnLine, 52(13), 1825-1830. https://doi.org/10.1016/j.ifacol.2019.11.501

Eggert, M., \& Alberts, J. (2020). Frontiers of business intelligence and analytics 3.0: a taxonomy-based literature review and research agenda. Business Research, 1-55. https://doi.org/10.1007/s40685-020-00108-y

García, S. G., \& García, M. G. (2019). Industry 4.0 implications in production and maintenance management: An overview. Procedia Manufacturing, 41, 415-422. https://doi.org/10.1016/j.promfg.2019.09.027

Goti, A., De la Calle, A., Gil, M. J., Errasti, A., Bom, P. R., \& García-Bringas, P. (2018). Development and application of an assessment complement for production system audits based 


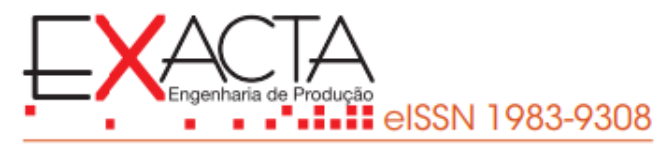

on data quality, IT infrastructure, and sustainability. Sustainability, 10(12), 4679. https://doi.org/10.3390/su10124679

Goti, A., Oyarbide-Zubillaga, A., Alberdi, E., Sanchez, A., \& Garcia-Bringas, P. (2019). Optimal Maintenance Thresholds to Perform Preventive Actions by Using Multi-Objective Evolutionary Algorithms. Applied Sciences, 9(15), 3068. https://doi.org/10.3390/app9153068 Hänel, T., \& Felden, C. (2017). Design and evaluation of an analytical framework to analyze and control production processes. Procedia CIRP, 62, 141-146. https://doi.org/10.1016/j.procir.2016.06.052

Jerman, A., Erenda, I., \& Bertoncelj, A. (2019). The influence of critical factors on business model at a smart factory: A case study. Business Systems Research Journal, 10(1), 42-52. DOI: 10.2478/bsrj-2019-0004

Mantravadi, S., \& Møller, C. (2019). An overview of next-generation manufacturing execution systems: how important is MES for industry 4.0?. Procedia manufacturing, 30, 588-595. https://doi.org/10.1016/j.promfg.2019.02.083

Nagy, J., Oláh, J., Erdei, E., Máté, D., \& Popp, J. (2018). The role and impact of Industry 4.0 and the internet of things on the business strategy of the value chain - the case of Hungary. Sustainability, 10(10), 3491. https://doi.org/10.3390/su10103491

Odważny, F., Wojtkowiak, D., Cyplik, P., \& Adamczak, M. (2019). Concept for measuring organizational maturity supporting sustainable development goals. LogForum, 15. DOI 10.17270/J.LOG.2019.321

Olszak, C. M., \& Mach-Król, M. (2018). A conceptual framework for assessing an organization's readiness to adopt big data. Sustainability, 10(10), 3734. https://doi.org/10.3390/su10103734.

Pereira, A. C., \& Romero, F. (2017). A review of the meanings and the implications of the $\begin{array}{llll}\text { Industry } & 4.0 & \text { concept. } & \text { Procedia }\end{array}$ https://doi.org/10.1016/j.promfg.2017.09.032.

Rikhardsson, P., \& Yigitbasioglu, O. (2018). Business intelligence \& analytics in management accounting research: Status and future focus. International Journal of Accounting Information Systems, 29, 37-58. https://doi.org/10.1016/j.accinf.2018.03.001.

Ruppert, T., Jaskó, S., Holczinger, T., \& Abonyi, J. (2018). Enabling technologies for operator 4.0: a survey. Applied sciences, 8(9), 1650. https://doi.org/10.3390/app8091650.

Saqlain, M., Piao, M., Shim, Y., \& Lee, J. Y. (2019). Framework of an IoT-based Industrial Data Management for Smart Manufacturing. Journal of Sensor and Actuator Networks, 8(2), 25. https://doi.org/10.3390/jsan8020025. 
Sayfouri, N. An alternative method of literature review: systematic review in english language teaching research. Procedia - Social and Behavioral Sciences, v.98, n.1, p.1693-1697, 2014. Soni, N., Sharma, E. K., Singh, N., \& Kapoor, A. (2020). Artificial Intelligence in Business: From Research and Innovation to Market Deployment. Procedia Computer Science, 167, 22002210. https://doi.org/10.1016/j.procs.2020.03.272

Schwab, K. (2016). A Quarta Revolução Industrial; Tradução Daniel Moreira Miranda, São Paulo, Edipro, ISBN 978-85-7283-978-5. http://creativecommons.org/licenses/by-nc-nd/4.0 Start - State of the Through Systematic Review, versão 3.4 Beta - LAPES - Laboratório de Pesquisa em Engenharia de Software - UFSCAR.

Tranfield, D., Denyer, D., \& Smart, P. (2003). Towards a methodology for developing evidence-informed management knowledge by means of systematic review. British journal of management, 14(3), 207-222. https://doi.org/10.1111/1467-8551.00375.

Reim, W., Parida, V. e Ortqvist, D., Product - Service Systems (PSS) business models and tactis - systematic literature review. Journal of Cleaner Production, v.97, n.1, p.61-75, 2015. https://doi.org/10.1016/j.jclepro.2014.07.003.

StArt. Software para apoio à Revisão Sistemática da Literatura. Desenvolvido pelo Laboratório LAPES-UFSCar. Disponível para download em: http://lapes.dc.ufscar.br/tools/start tool. Acessado em: março/2020.

\section{AGRADECIMENTOS}

"O presente trabalho foi realizado com apoio da Coordenação de Aperfeiçoamento de Pessoal de Nível Superior - Brasil (CAPES) - Código de Financiamento 001”.

"This study was financed in part by the Coordenação de Aperfeiçoamento de Pessoal de Nível Superior - Brasil (CAPES) - Finance Code 001"

(Portaria n ${ }^{\circ}$ 206, 04/09/2018). 